\title{
Assessment of task-based image quality for abdominal CT protocols linked with national diagnostic reference levels
}

\author{
Anaïs Viry ${ }^{1}$ (D) $\cdot$ Christoph Aberle ${ }^{2} \cdot$ Thiago Lima $^{1,3} \cdot$ Reto $^{\text {Treier }}{ }^{4} \cdot$ Sebastian T. Schindera ${ }^{5}$. Francis R. Verdun ${ }^{1}$. \\ Damien Racine ${ }^{1}$
}

Received: 11 January 2021 /Revised: 4 June 2021 / Accepted: 29 June 2021 / Published online: 29 July 2021

(C) The Author(s) 2021

\begin{abstract}
Objectives To assess task-based image quality for two abdominal protocols on various CT scanners. To establish a relationship between diagnostic reference levels (DRLs) and task-based image quality.

Methods A protocol for the detection of focal liver lesions was used to scan an anthropomorphic abdominal phantom containing 8and 5-mm low-contrast $(20 \mathrm{HU})$ spheres at five $\mathrm{CTDI}_{\mathrm{vol}}$ levels $(4,8,12,16$, and $20 \mathrm{mGy})$ on 12 CTs. Another phantom with highcontrast calcium targets (200 HU) was scanned at 2, 4, 6, 10, and $15 \mathrm{mGy}$ using a renal stones protocol on the same CTs. To assess the detectability, a channelized Hotelling observer was used for low-contrast targets and a non-prewhitening observer with an eye filter was used for high contrast targets. The area under the ROC curve and signal to noise ratio were used as figures of merit.

Results For the detection of 8-mm spheres, the image quality reached a high level (mean AUC over all CTs higher than 0.95 ) at $11 \mathrm{mGy}$. For the detection of 5-mm spheres, the AUC never reached a high level of image quality. Variability between CTs was found, especially at low dose levels. For the search of renal stones, the AUC was nearly maximal even for the lowest dose level. Conclusions Comparable task-based image quality cannot be reached at the same dose level on all CT scanners. This variability implies the need for scanner-specific dose optimization.

Key Points

- There is an image quality variability for subtle low-contrast lesion detection in the clinically used dose range.

- Diagnostic reference levels were linked with task-based image quality metrics.

- There is a need for specific dose optimization for each CT scanner and clinical protocol.
\end{abstract}

Keywords Multidetector computed tomography $\cdot$ Diagnostic reference levels $\cdot$ Radiation protection

Anaïs Viry and Christoph Aberle contributed equally to this work.

Anaiis Viry

Anais.viry@chuv.ch

1 Institute of Radiation Physics, Lausanne University Hospital and University of Lausanne, Lausanne, Switzerland

2 Department of Radiology, University Hospital Basel, University of Basel, Basel, Switzerland

3 Department of Radiology and Nuclear Medicine, Kantonsspital Luzern, Lucerne, Switzerland

4 Radiological Protection Division, Federal Office of Public Health, Bern, Switzerland

5 Department of Radiology, Kantonsspital Aarau, Aarau, Switzerland

\begin{tabular}{|c|c|}
\hline \multicolumn{2}{|c|}{ Abbreviations } \\
\hline AUC & Area under the ROC curve \\
\hline $\mathrm{CHO}$ & Channelized Hotelling observer \\
\hline $\mathrm{CTDI}_{\mathrm{vol}}$ & Volume computed tomography dose index \\
\hline $\mathrm{CTDI}_{\mathrm{w}}$ & Weighted computed tomography dose index \\
\hline DDoG & Dense difference of Gaussians \\
\hline DRL & Diagnostic reference level \\
\hline HU & Hounsfield units \\
\hline IR & Iterative reconstruction \\
\hline NPS & Noise power spectrum \\
\hline NPWE & Non-prewhitening observer with an eye filter \\
\hline ROI & Region of interest \\
\hline SNR & Signal-to-noise ratio \\
\hline TTF & Target transfer function \\
\hline
\end{tabular}




\section{Introduction}

The contribution of computed tomography (CT) to the total effective dose due to medical X-ray examinations has been recently reported to be up to $70 \%$ [1]. Hence, continuous efforts have been made by manufacturers and users of CT to reduce the dose level per examination with the integration of new technologies (e.g., tube current modulation, iterative reconstruction (IR) algorithms, or more efficient detectors) and the optimization of clinical protocols [2].

An important aspect to take into account when dealing with protocol optimization is the variation of the practice even for a well-defined indication. Hence, diagnostic reference levels (DRLs) were proposed by the International Commission on Radiological Protection (ICRP) in 1996 to reduce the variability of clinical practice by leading users of $\mathrm{CT}$ to take actions when the local dose indicator systematically exceeds the national DRL [3]. Two major limitations appear. DRLs are often not related to precise clinical indication, nor to any clinical image quality criteria. The first limitation was partially addressed by recently published national or local DRLs [4-9], and at the European level [10]; the second one is still an open question as mentioned by Rehani [11]. Moreover, the technological differences between CT scanners should be taken into account when dealing with clinical protocol optimization. Adjusting the radiation dose level of a clinical protocol using the value of the associated DRL without assessing the image quality is suboptimal [12]. On the one hand, further patient dose optimization could be justified for the most modern CT scanners. On the other hand, it could cause an excessive dose reduction with a loss of diagnostic performance, in particular for older CT scanners. This practice can lead to variations in image quality and patient care, while the goal is the standardization of image quality such that it is just sufficient for the clinical task at the lowest possible dose [13, 14]. Hence, it appears necessary to associate national DRLs for specific clinical tasks with task-based image quality criteria in order to assess a potential dose optimization and avoid excessive patient dose reduction.

Among all existing CT examinations, abdominal CT protocols deliver the highest radiation doses to the patients [1]. Moreover, the optimization process is particularly crucial for abdominal protocols due to the challenges arising from the detection of small low-contrast lesions [15]. An excessive patient dose reduction can highly increase the risk of missing subtle lesions.

The use of basic image quality metrics (standard deviation, contrast, contrast-to-noise ratio, modulation transfer function) is of limited interest because they are not directly related to any clinical requirement [16]. Task-based image quality analysis was initially proposed by Barrett and Myers to quantify the CT diagnostic performances $[17,18]$. The methodology was recently applied with success to benchmark CT scanners
[19] and clinical protocols [20] or assess the use of IR algorithms $[16,21]$.

The purpose of this contribution is to assess task-based image quality for two abdominal protocols on various CT scanners and to establish a relationship between DRL values and image quality for the respective clinical tasks.

\section{Materials and methods}

\section{Image quality phantoms}

An abdominal anthropomorphic phantom (QRM, A PTW COMPANY) was used to assess the image quality of two examination types. The phantom mimics various tissues (muscle, liver, spleen, and vertebrae) (Fig. 1a). Due to the absence of materials with high atomic numbers, the phantom was designed to assess non-contrast CT scans. Its effective diameter of $30 \mathrm{~cm}$ simulates the attenuation of a patient with a weight around $75 \mathrm{~kg}$. The phantom contains a hole of $10 \mathrm{~cm}$ in diameter into which different modules can be inserted. To mimic the detection of focal liver lesions, a first module containing hypodense low-contrast spheres of different sizes (in particular 8 and $5 \mathrm{~mm}$ diameter) with a contrast of $20 \mathrm{HU}$ relative to the background was used (Fig. 1b). These two lesion sizes were considered clinically relevant. Indeed, liver lesions smaller than $5 \mathrm{~mm}$ are often benign. Furthermore, it is difficult to accurately characterize smaller lesion sizes in the liver with this type of contrast in CT [22].

A second module containing a high contrast calcic rod of $20 \mathrm{~mm}$ in diameter and a contrast of $200 \mathrm{HU}$ was used to quantify the spatial resolution, an important aspect for assessing the detection of renal stones (Fig. 1c).

\section{CT scanners and acquisition/reconstruction parameters}

In concertation with a panel of radiologists, two sets of acquisition and reconstruction parameter settings were defined that are typical for examinations of a) focal liver lesions and b) renal stones. Five volume computed tomography dose index $\left(\mathrm{CTDI}_{\mathrm{vol}}\right)$ levels were used for each set $(4,8,12,16$, and $20 \mathrm{mGy}$ for focal liver lesions and 2, 4, 6, 10, and $15 \mathrm{mGy}$ for renal stones). The current Swiss DRLs (11 mGy for focal liver lesions $\mathrm{CT}$ acquisitions and $6 \mathrm{mGy}$ for renal stones $\mathrm{CT}$ acquisitions) and the underlying dose distributions [6] were used to determine the $5 \mathrm{CTDI}_{\mathrm{vol}}$ levels, so that they cover the clinically relevant dose range.

The 12 CT scanners involved in this study are listed in Table 1. Three different CT scanners from each of the four major CT manufacturers were included. Thus, the variability of image quality due to scanner-specific technology properties 


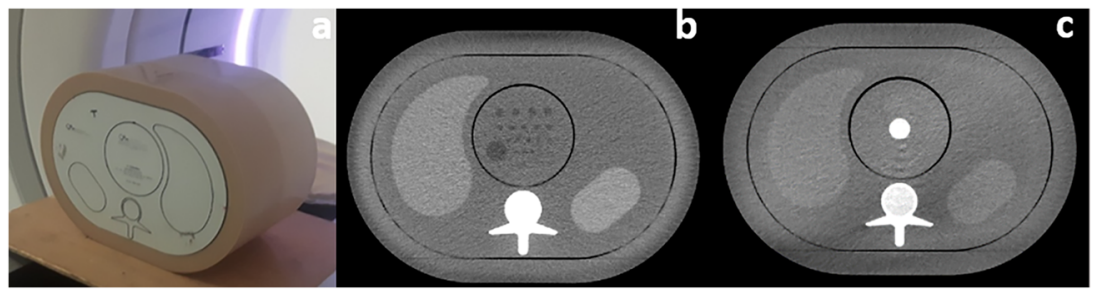

Fig. 1 a Photo of the QRM phantom. b CT slice of the phantom with the module containing the low-contrast spheres, the $8-\mathrm{mm}$ spheres are all positioned on the first row and the $5-\mathrm{mm}$ spheres are positioned on the

could be adequately studied. In practice, there is no identical set of acquisition and reconstruction parameters that can be used on all CT scanner models. Instead, acquisition and reconstruction parameters were matched as closely as possible (Table 1). Reconstruction algorithms and reconstruction kernels are manufacturer- and model-specific.

\section{Radiation dose assessment}

Before each acquisition session, $\mathrm{CTDI}_{\mathrm{w}}$ was measured with a 10-cm ionization chamber (PTW TM30009 or Radcal 10X63CT) using a 32-cm-diameter CTDI phantom, following the international electrotechnical commission (IEC) standard 60601-2-44. The ratio of the measured $\mathrm{CTDI}_{\mathrm{w}}$ to the displayed $\mathrm{CTDI}_{\mathrm{w}}$ was used to correct the displayed $\mathrm{CTDI}_{\mathrm{vol}}$ of the image quality phantom scans. For the $12 \mathrm{CT}$ scanners, the correction factors ranged from 0.847 to 1.057 . Furthermore, the actual radiation dose depends on the $\mathrm{z}$ position if the tube current is modulated. All CTDI $\mathrm{vol}_{\mathrm{vl}}$ values presented in the results section are corrected and refer to the actual z-position where the image quality was evaluated.

Relative standard uncertainties on the final $\mathrm{CTDI}_{\mathrm{vol}}$ values were evaluated in detail [23]. It turned out that $2.5 \%$ is a good estimate for all CT scanners and all dose levels. The most important uncertainty component was the uncertainty of the $\mathrm{CTDI}_{\mathrm{w}}$ measurements, more specifically the uncertainty of the chamber calibration factors (relative standard uncertainty of $1.5 \%$, from calibration certificate).

\section{Image analysis}

\section{Low-contrast detectability}

We quantitatively assessed the image quality using a taskbased methodology. The clinical tasks were the detection of low contrast lesions with a size of 5 and $8 \mathrm{~mm}$. The lowcontrast module contains four spheres of $8 \mathrm{~mm}$ and five spheres of $5 \mathrm{~mm}$ in diameter in the exact same slice. As 20 acquisitions for each dose level were acquired, we were able to extract at least 80 square regions of interest (ROIs) of $18 \times$ 18 pixels containing lesions of $8 \mathrm{~mm}$ and $5 \mathrm{~mm}$ in diameter. On the right homogeneous part of the phantom images, 400 third row. $\mathbf{c}$ CT slice of the phantom with the module containing the high contrast rod

ROIs containing only noise were extracted in five slices around the slice of interest (Fig. 1b).

An anthropomorphic mathematical model observer was chosen to quantitatively assess the detectability of low contrast lesions. Based on Bayesian statistical decision theory, this kind of observer has the ability to mimic human observer responses in the detection of low contrast structures in an image [24-26]. The channelized Hotelling observer (CHO) with 10 dense difference of Gaussian channels (DDoG) was applied, following the methodology proposed by Wunderlich et al to compute the signal-to-noise ratio (SNR), expressing the detectability of the lesion [27]. The CHO model observer was previously computed using the same anthropomorphic phantom [21]. As CHO model observers are more efficient than human observers for simple detection tasks in uniform background, it is necessary to adjust the detection outcomes of model observers by adding internal noise on the covariance matrix [28]. Internal noise was calibrated with the data from the inter-comparison study of $\mathrm{Ba}$ et al [29]. The area under the receiver operating characteristics curve (AUC) was used as the figure of merit to assess the detectability of low contrast lesions. A monotonic function can link SNR and AUC [30]. The AUC was computed for each $\mathrm{CT}$, dose level, and lesion size.

\section{High-contrast detectability}

For the detection of renal stones, we also used a task-based methodology. The clinical task was the detection of calcic lesions of 3 and $5 \mathrm{~mm}$ with a contrast of $450 \mathrm{HU}$. Indeed, renal stones of $3 \mathrm{~mm}$ and smaller have a high chance of spontaneous passage [31]. We decided to use $3 \mathrm{~mm}$ as a cut-off. An anthropomorphic mathematical observer, the nonprewhitening observer with an eye filter (NPWE) expressed in the Fourier domain was used. Developed by Burgess [32], the NPWE computes the SNR of simulated high contrast lesions using the in-plane contrast-dependent spatial resolution (target transfer function (TTF)) from the images of high contrast objects, the noise power spectrum (NPS), and the virtual transfer function of the human eye [33].

The TTF was computed using the module containing the high-contrast rod. As six acquisitions were performed for each CT scanner and dose level, 78 ROIs of $64 \times 64$ pixels centered 


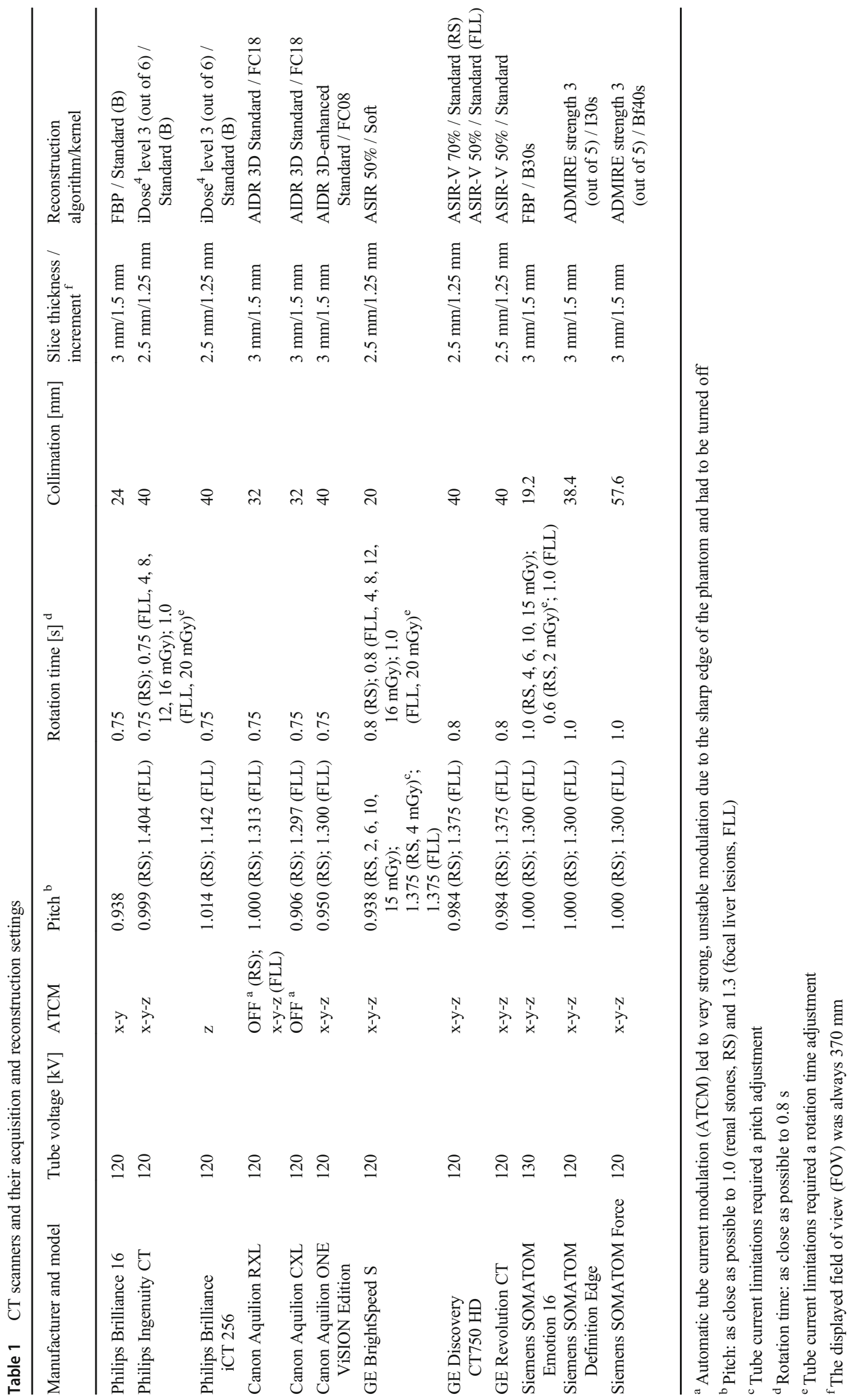


on the rod could be extracted. The 2D TTF was calculated from the edge of the rod following the methodology described by Monnin et al and radially averaged and normalized at the zero frequency to obtain the 1D TTF [34].

Image noise was quantified by computing the NPS [35-37]. A total of 90 ROIs of $64 \times 64$ pixels were extracted from 15 homogeneous slices per acquisition. The 2D NPS was computed on the cropped ROIs and then radially averaged to obtain 1D NPS.

As the integral of 1D NPS decreases as the slice thickness increases [38], we corrected the SNR of the NPWE model for the $5 \mathrm{CT}$ scanners with a $2.5 \mathrm{~mm}$ slice thickness by a factor $\sqrt{\frac{3}{2.5}}$.

\section{Statistical analysis}

For the CHO model observer, to reduce the positive bias caused by the use of a finite number of images and to compute the exact 95\% confidence interval of SNR, the methodology developed by Wunderlich was applied [27]. A linear fit between the logarithm of the SNR and the logarithm of the dose, taking into account the uncertainties, was performed for each CT scanner to calculate SNR and AUC values at a given $\mathrm{CTDI}_{\mathrm{vol}}$ and vice versa.
For the NPWE outcome, the uncertainties were determined using a bootstrap method. Results were computed using 100 bootstrapped samples of 50 ROIs used for TTF and NPS calculations.

\section{Results}

To ensure the impartiality of this work, the results are reported in an anonymous manner consistently throughout the manuscript. It was not the purpose of this work to compare individual CT scanner models but rather to study the size of the variability when using different models. A capital letter (A, $\mathrm{B}, \mathrm{C}$, and D) was assigned to each manufacturer and figures 1 , 2 , and 3 refer to the three different $\mathrm{CT}$ scanners.

\section{Low-contrast detectability}

As expected, irrespective of the lesion size, the low contrast detectability increased with the dose level (Figs. 2 and 3).

For the largest lesion size $(8 \mathrm{~mm})$, at $11 \mathrm{mGy}$, corresponding to the Swiss DRL of the investigated liver protocol, the AUC reached a high image quality level with values higher than 0.95 for 10 out of 12 CT scanners (Fig. 2 and Table 2). The use of a dose level below $7 \mathrm{mGy}\left(25^{\text {th }}\right.$ percentile of the DRL distribution) induced a loss of image quality. The

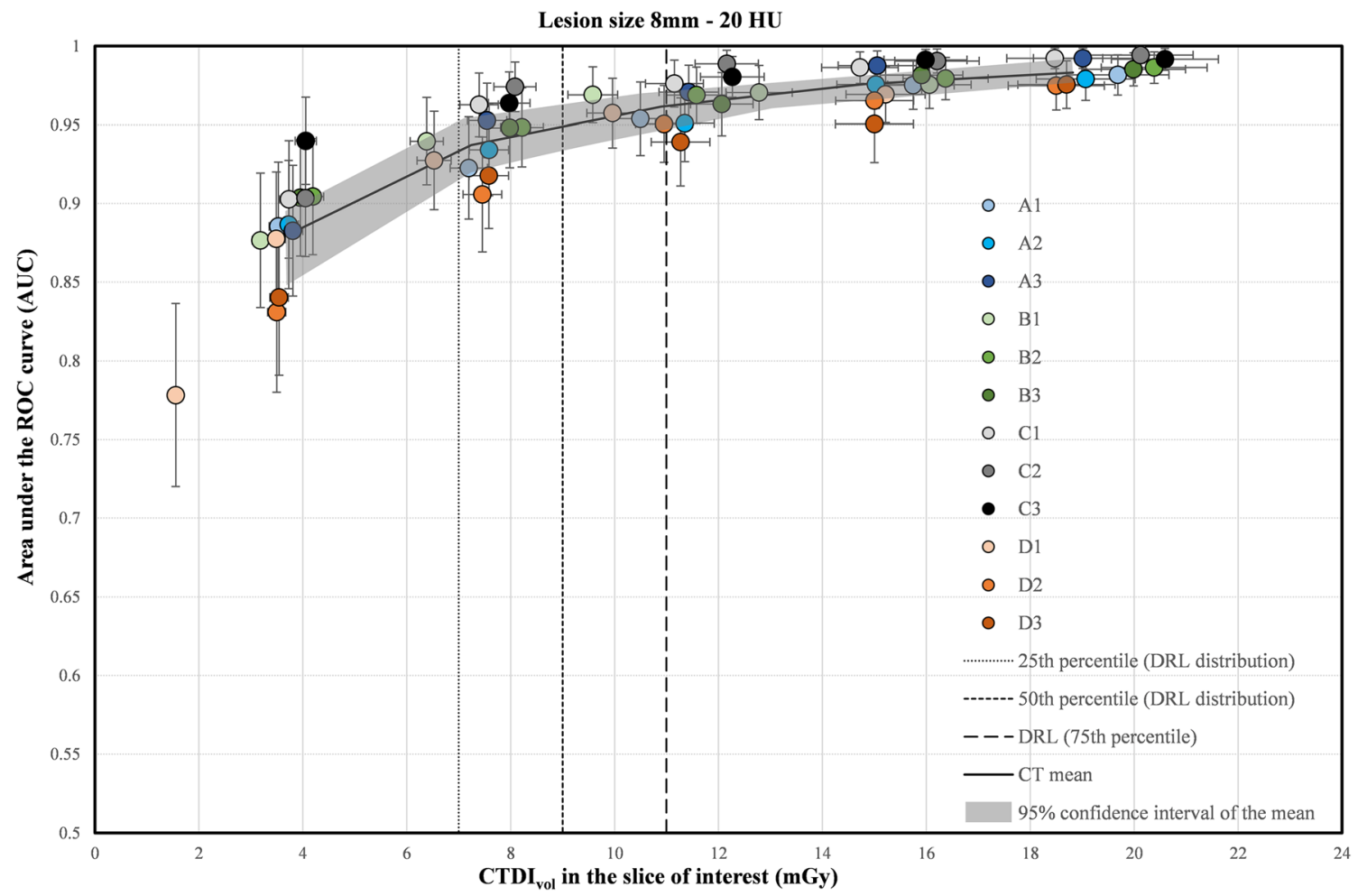

Fig. 2 Area under the ROC curve as a function of $\mathrm{CTDI}_{\mathrm{vol}}$ in the slice of interest for the 8-mm lesion size for the $12 \mathrm{CT}$ scanners. The horizontal and vertical uncertainty bars represent the expanded uncertainty $(\mathrm{k}=2$, 95\% level of confidence) for the $\mathrm{CTDI}_{\mathrm{vol}}$ and AUC, respectively. The solid black line was plotted by joining 5 points representing the mean AUC and the mean CTDI ${ }_{\mathrm{vol}}$ over all $12 \mathrm{CT}$ s for each dose level. The gray band was plotted by joining the limits of the $95 \%$ confidence intervals of the 5 points 


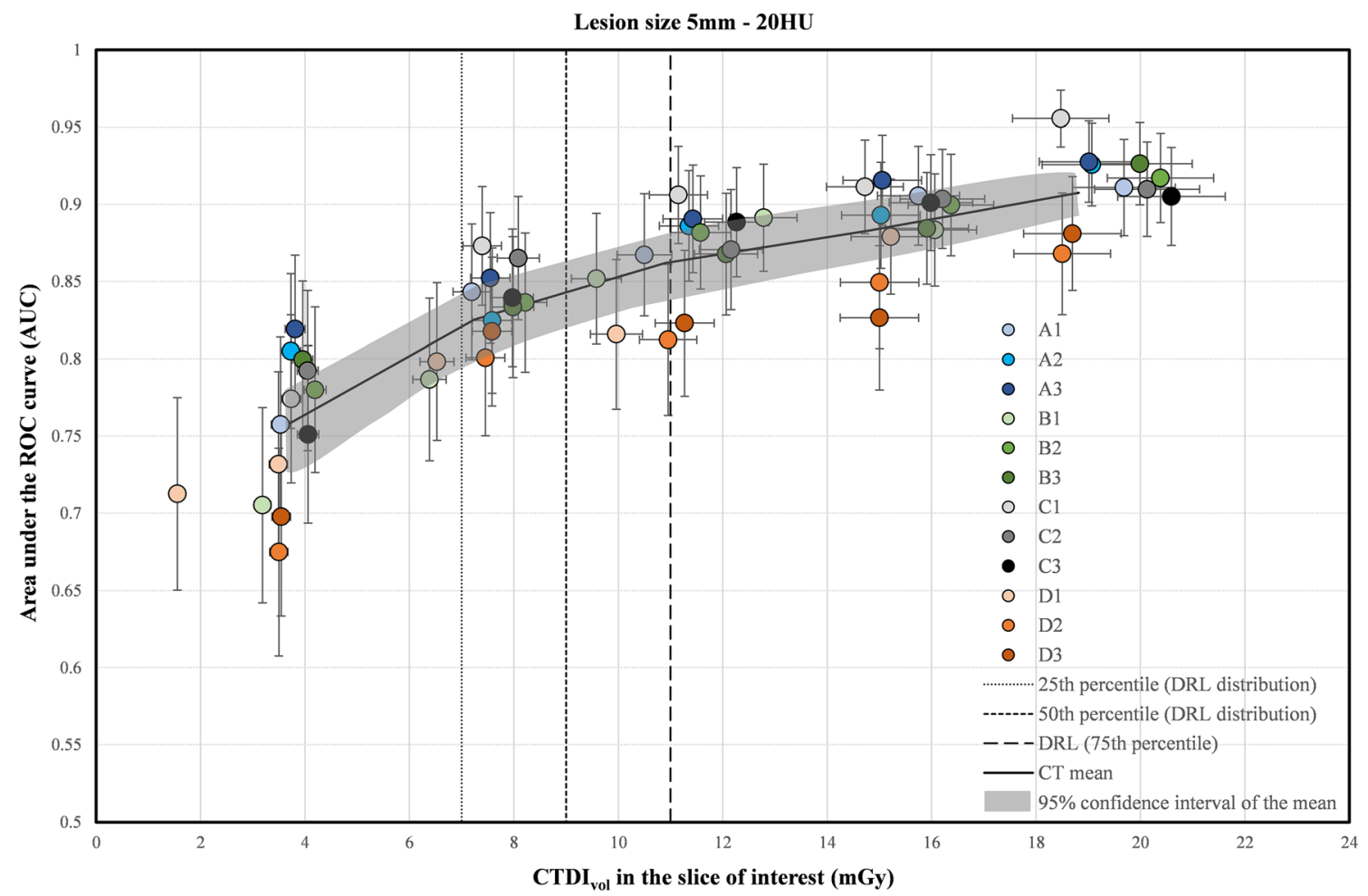

Fig. 3 Area under the ROC curve as a function of CTDI $\mathrm{vol}_{\mathrm{vol}}$ in the slice of interest for the 5-mm lesion size for the $12 \mathrm{CT}$ scanners. The horizontal and vertical uncertainty bars represent the expanded uncertainty $(\mathrm{k}=2$, 95\% level of confidence) for the $\mathrm{CTDI}_{\mathrm{vol}}$ and AUC, respectively. The

percentage of AUC reduction when decreasing the dose level from 11 to $7 \mathrm{mGy}$, varied from 1.7 to $4.3 \%$ for the various CT scanners. The variability of image quality between the various CTs is higher at low-dose levels: The AUC ranged from 0.90 to 0.96 at $7 \mathrm{mGy}$ (Table 2). A comparable level of image quality was obtained at substantially different $\mathrm{CTDI}_{\mathrm{vol}}$ values. For example, an AUC of 0.95 was obtained at a range of doses between 5.3 and $13 \mathrm{mGy}$, as calculated using the best-fit equations.

For the smaller lesion size $(5 \mathrm{~mm})$, the AUC results were lower than for the $8 \mathrm{~mm}$ lesion size, as expected. The AUC increased with the dose but never reached a high level of image quality for all CTs. Indeed, the mean AUC over all CT scanners was only 0.86 at $11 \mathrm{mGy}$ and reached 0.91 for the highest dose level (Fig. 3). The use of a dose level lower than the DRL induced a higher loss of image quality in comparison with the 8-mm lesion size (Table 2). The percentage of AUC reduction when decreasing the dose level from 11 to 7 mGy, varied from 3.6 to $6.8 \%$ for the various CTs. The AUC ranged from 0.76 to 0.86 at $7 \mathrm{mGy}$. An AUC of 0.85 was obtained at a range of doses between 6.0 and $14.3 \mathrm{mGy}$.

\section{High-contrast detectability}

The most challenging high contrast task was the detection of a $3 \mathrm{~mm}$ calcic lesion (Fig. 4). The results for the 5-mm lesion are solid black line was plotted by joining 5 points representing the mean AUC and the mean CTDI $_{\mathrm{vol}}$ over all $12 \mathrm{CTs}$ for each dose level. The gray band was plotted by joining the limits of the $95 \%$ confidence intervals of the 5 points

presented in Fig. 5. For each CT, the detectability increased with the dose. But even at the lowest dose level ( $2 \mathrm{mGy})$, for both lesion sizes, the SNR for all CTs was very high (AUC close to 1.0), indicating that the detection of lesions with such sizes and nominal contrast relative to a homogeneous background was trivial.

\section{Discussion}

In the framework of patient radiation dose optimization, it is essential to ensure that both the dose and image quality are equally balanced to fulfill the diagnostic requirements at the lowest possible dose [13]. The detection of low-contrast lesions in a uniform background is a simple task in comparison with the complexity of a radiological diagnosis for the detection of focal liver lesions. However, even in this simple condition, the task is challenging (Figs. 2 and 3). For the largest lesion size investigated $(8 \mathrm{~mm})$, the dose optimization curve reaches a high level of image quality (mean AUC over all CTs higher than 0.95) at approximately $11 \mathrm{mGy}$ (corresponding to the DRL). However, there is a loss of low-contrast detectability for all CTs when using lower dose levels. Our results indicate that one has to be cautious when using doses below the current Swiss DRL (11 mGy) and even more below the $25^{\text {th }}$ percentile (7 mGy), as discussed in ICRP 135 [12]. For 


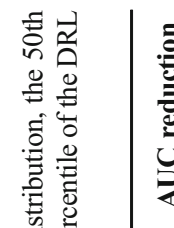

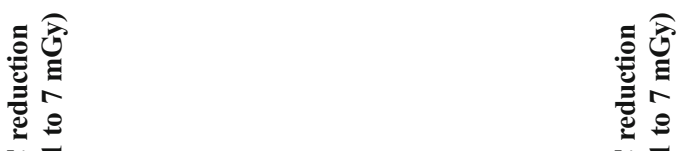

$\because$

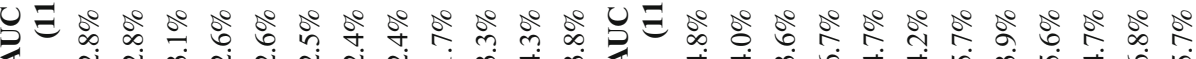

름

胥

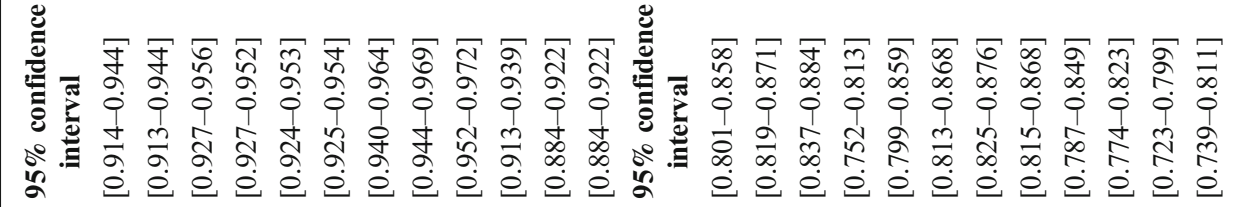

离,

究蓉

ક

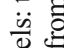

可

要

证.

产

$\frac{\widehat{n}}{\frac{0}{0}}$

过

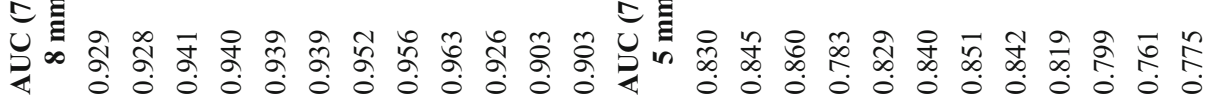

蕰

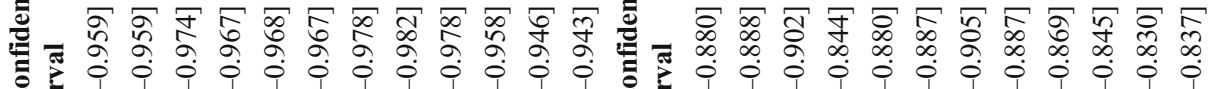

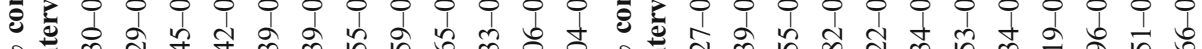

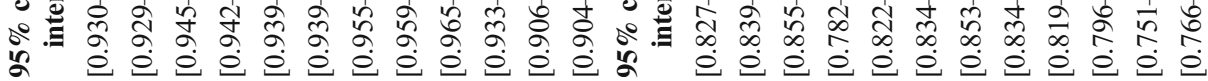

害

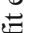

$\stackrel{0}{\rightleftarrows}$

:

莺

尝

:

范

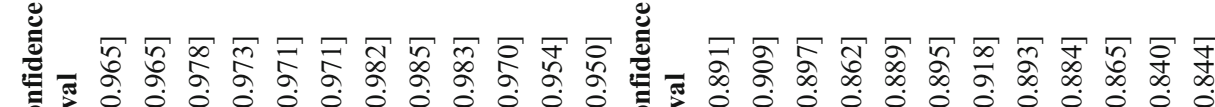

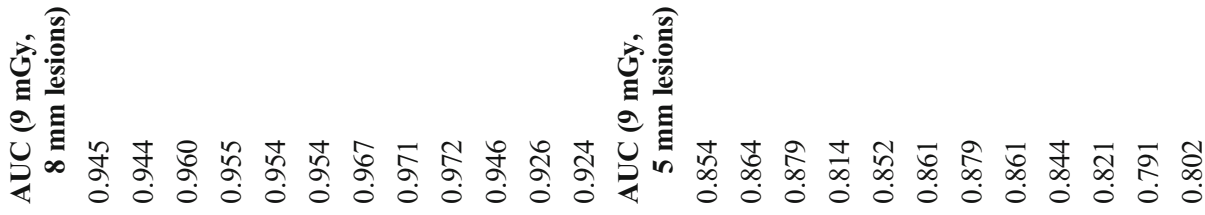




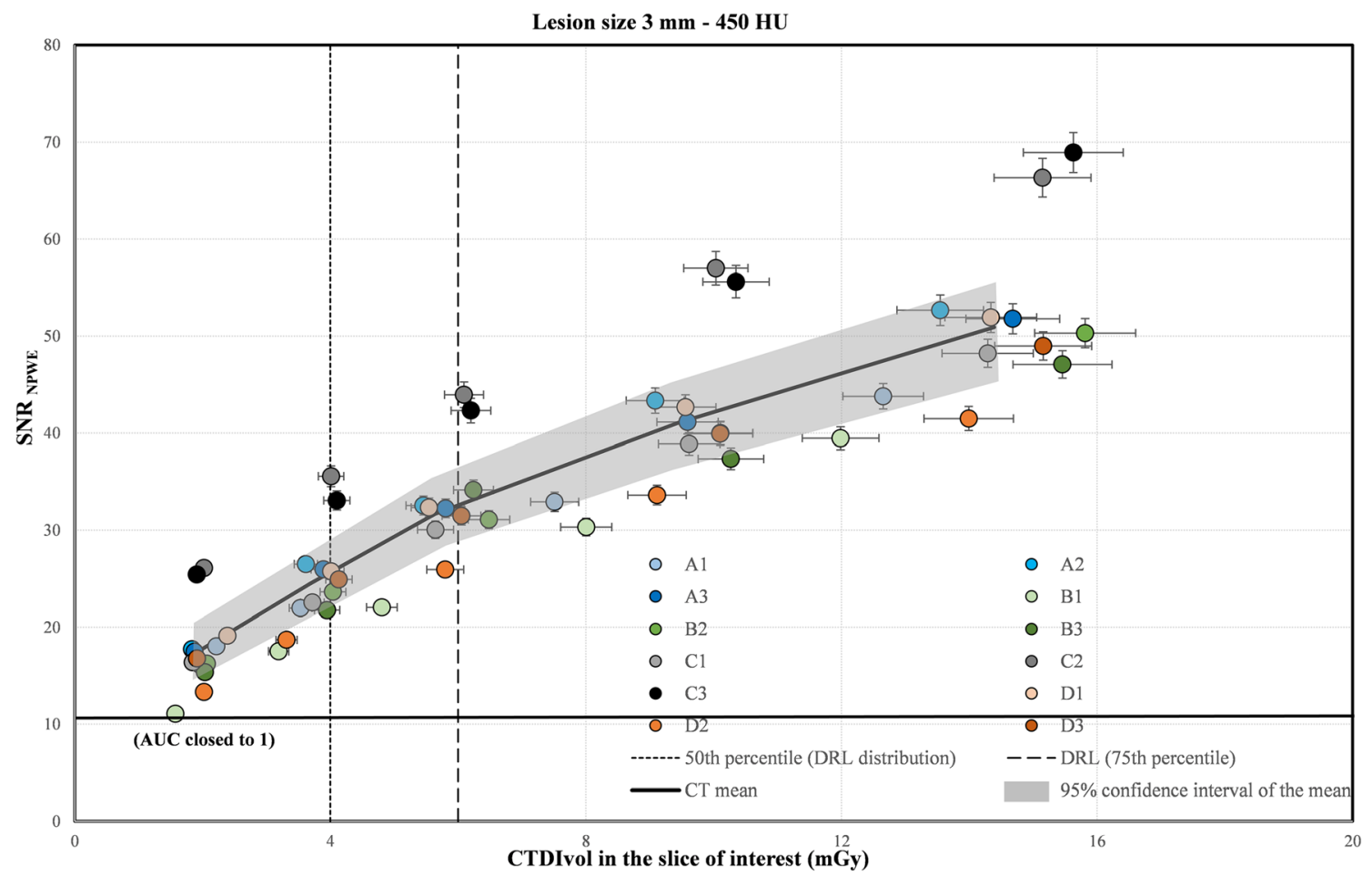

Fig. 4 Signal-to-noise ratio (SNR) calculated for the 3-mm lesion size using the NPWE model observer as a function of $\mathrm{CTDI}_{\mathrm{vol}}$ in the slice of interest for the $12 \mathrm{CT}$ scanners. The vertical and horizontal uncertainty bars represent the expanded uncertainty $(\mathrm{k}=2,95 \%$ level of confidence) for the SNR and the $\mathrm{CTDI}_{\mathrm{vol}}$, respectively. The solid black line was plotted by joining 5 points representing the mean SNR and the mean $\mathrm{CTDI}_{\mathrm{vol}}$ over all $12 \mathrm{CTs}$ for each dose level. The gray band was plotted by joining the limits of the $95 \%$ confidence intervals of the 5 points

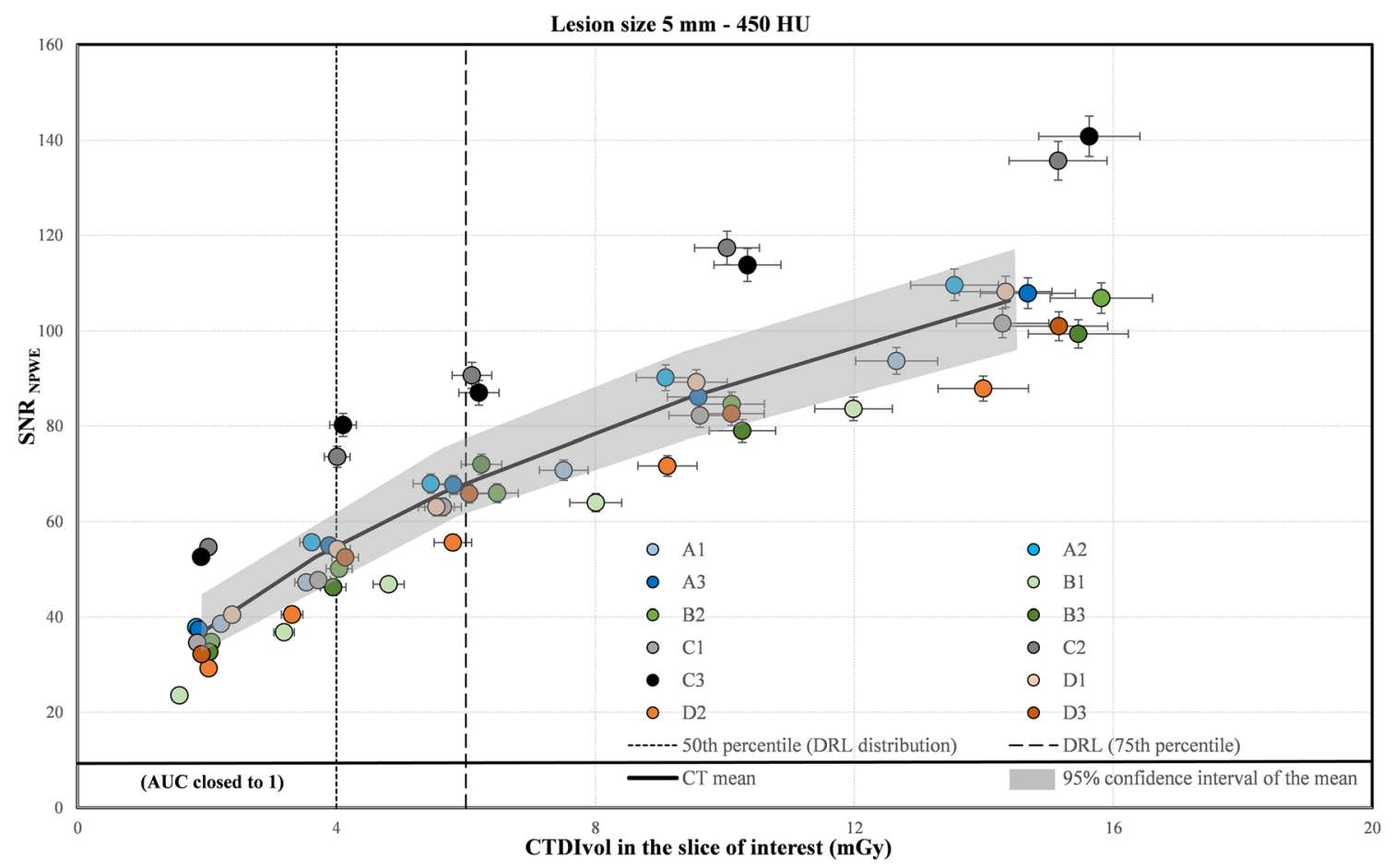

Fig. 5 Signal-to-noise ratio (SNR) calculated for the $5 \mathrm{~mm}$ lesion size using the NPWE model observer as a function of $\mathrm{CTDI}_{\mathrm{vol}}$ in the slice of interest for the $12 \mathrm{CT}$ scanners. The vertical and horizontal uncertainty bars represent the expanded uncertainty $(\mathrm{k}=2,95 \%$ level of confidence) for the SNR and the $\mathrm{CTDI}_{\mathrm{vol}}$, respectively. The solid black line was plotted by joining 5 points representing the mean SNR and the mean $\mathrm{CTDI}_{\mathrm{vol}}$ over all $12 \mathrm{CTs}$ for each dose level. The gray band was plotted by joining the limits of the $95 \%$ confidence intervals of the 5 points 
the 5-mm lesion size, the task is even more challenging. The detectability never reached a high level of image quality when increasing the dose from 4 to $20 \mathrm{mGy}$. Furthermore, the variations in image quality between CT scanners should imply a difference of diagnostic information contained in clinical images. Conversely, different doses should be used to achieve the same outcome when dealing with low contrast detection (see Table 2). This shows the limitation of the DRL concept for optimizing radiation dose without assessing image quality. The high contrast detection task was chosen to simulate the detection of renal stones. It appears that this task in homogeneous background is not challenging enough to assess the potential dose optimization. Even for the smallest dose level investigated $(2 \mathrm{mGy})$ and the smallest lesion size $(3 \mathrm{~mm}$ in diameter), the detectability is very high for all CTs, indicating a perfect detection in this simple condition. Nevertheless, differences in the SNR between the CT scanners were observed for all five dose levels (Fig. 4). With these results, it seems reasonable to hypothesize that correct optimization would lead to different doses on different $\mathrm{CT}$ scanners for a more realistic, more challenging high-contrast detection task with anatomical background, or for size, shape, and CT number determination.

The results show that it is necessary to link national DRLs for specific clinical tasks with task-based image quality criteria. In the future, an image quality reference level associated with the DRL could be used for specific clinical tasks [39]. A discussion among the radiologists, the community should also be initiated to define a minimum level of image quality required, depending on the clinical indications, for a safe diagnosis. This could avoid excessive patient dose reduction, in particular for the detection of subtle lesions, as reported by several authors in phantoms [40, 41] and also in patient studies [42]. This approach follows ICRP publication 135, claiming that the "application of DRL values is not sufficient for optimization of protection. Image quality must be evaluated as well" [12]. The assessment of task-based image quality using mathematical observers is an objective and quantitative approach [17] and the outcomes are linked with human observer performances [26, 39]. The phantom presents some limitations. Firstly, the contrast of the various lesions in the phantom was created using plastic materials of low atomic numbers and cannot perfectly simulate the contrast of lesions in a CT acquisition that uses a contrast agent. Ideally, a phantom with iodine lesions should be used to optimize arterial and venous phases of abdominal protocols. Secondly, the background was homogeneous. We should expect that the use of a realistic anatomical background would be more challenging and the AUC results would be worse [43]. CT scanner-specific settings and properties like collimation, flying focus technique, pitch, tube voltage, rotation time, ATCM settings, reconstruction algorithms, slice thickness, and increment are not identical. However, these differences cannot be avoided. Particularly, the 3-mm slice thickness with an increment of $1.5 \mathrm{~mm}$ is not optimal to minimize the partial volume effect of the $5 \mathrm{~mm}$ lesion size [44]. Moreover, we did not reposition the phantom between scans, so the effect was not averaged out. Furthermore, the standard IEC CTDI ${ }_{w}$ measurement method that was used in this study is known to underestimate $\mathrm{CTDI}_{\mathrm{w}}$ for wide $\mathrm{CT}$ beams because the scatter equilibrium is not achieved [45, 46]. However, no correction factor was applied to the IEC measurements because the collimation was smaller than $40 \mathrm{~mm}$ for 11 out of 12 CTs [47]. The described differences in CT scanner specific settings and properties do not allow a completely fair comparison between scanners. However, the goal was not to rate the CT scanners but to study typical CT scanner variability of the image quality at a given dose. Despite the stated limitations, the results show the limitation of the DRL concept. Hence, CT scanner modelspecific DRLs could be an option to avoid an unjustified wide dispersion of image quality for well-defined clinical tasks. However, due to the great diversity of CT models and manufacturers on the market, their implementation in clinical routine is difficult. The application of local DRLs to check the clinical practice may be easier to implement using Dose Archiving and Communication Systems (DACS). Ideally, dose optimization should encompass both the DRL process and image quality evaluation using a task-based paradigm. However, the highest priority for the optimization process is to ensure that the image quality is sufficient for the clinical question.

In conclusion, task-based image quality was assessed for various dose levels related to the current DRL values. Assessing image quality metrics related to the clinical question to be answered must be an important part of the optimization process. Comparable image quality for specific clinical questions cannot be reached at the same dose level on all CT scanners. This variability between CTs implies the need for a CT model-specific dose optimization.

Acknowledgements The authors gratefully acknowledge the support from the following participating radiological institutes in data collection: Centre Hospitalier Universitaire Vaudois (CHUV), Centre d ' Imagerie Diagnostique de Lausanne, Felix Platter Spital Basel, Kantonsspital Aarau, Kantonsspital Baselland, Schmerzklinik Basel, Spital Altstätten (Spitalregion Rheintal Werdenberg Sarganserland), Universitätsspital Basel.

Funding Open Access funding provided by Université de Lausanne. This study has received funding from the Swiss Federal Office of Public Health (FOPH), contract number 17.017358.

\section{Declarations}

Guarantor The scientific guarantor of this publication is Dr. Damien Racine.

Conflict of interest The authors of this manuscript declare no relationships with any companies, whose products or services may be related to the subject matter of the article. 
Statistics and biometry One of the authors has significant statistical expertise.

Informed consent Not applicable.

Ethical approval Institutional Review Board approval was not required because the article only deals with medical imaging phantoms.

\section{Methodology}

- experimental

- multicenter study

Open Access This article is licensed under a Creative Commons Attribution 4.0 International License, which permits use, sharing, adaptation, distribution and reproduction in any medium or format, as long as you give appropriate credit to the original author(s) and the source, provide a link to the Creative Commons licence, and indicate if changes were made. The images or other third party material in this article are included in the article's Creative Commons licence, unless indicated otherwise in a credit line to the material. If material is not included in the article's Creative Commons licence and your intended use is not permitted by statutory regulation or exceeds the permitted use, you will need to obtain permission directly from the copyright holder. To view a copy of this licence, visit http://creativecommons.org/licenses/by/4.0/.

\section{References}

1. Viry A, Bize J, Trueb PR et al (2021) Annual exposure of the Swiss population from medical imaging In 2018. Radiat Prot Dosim. https://doi.org/10.1093/rpd/ncab012

2. Kalra MK, Maher MM, Toth TL et al (2004) Techniques and applications of automatic tube current modulation for CT. Radiology 233:649-657

3. ICRP (1996) Radiological protection and safety in medicine. Pergamon, Oxford

4. Lajunen A (2015) Indication-based diagnostic reference levels for adult CT-examinations in Finland. Radiat Prot Dosim 165:95-97

5. Habib Geryes B, Hornbeck A, Jarrige V, Pierrat N, Ducou Le Pointe H, Dreuil S (2019) Patient dose evaluation in computed tomography: a French national study based on clinical indications. Phys Med 61:18-27

6. Aberle C, Ryckx N, Treier R, Schindera S (2020) Update of national diagnostic reference levels for adult CT in Switzerland and assessment of radiation dose reduction since 2010. Eur Radiol 30: 1690-1700

7. Brat H, Zanca F, Montandon S et al (2019) Local clinical diagnostic reference levels for chest and abdomen $\mathrm{CT}$ examinations in adults as a function of body mass index and clinical indication: a prospective multicenter study. Eur Radiol 29:6794-6804

8. Public Health England (2019) National diagnostic reference levels (NDRLs). Available via https://www.gov.uk/government/ publications/diagnostic-radiology-national-diagnosticreferencelevels-ndrls/ndrl. Accessed 24 Mar 2021

9. Schegerer A, Loose R, Heuser LJ, Brix G (2019) Diagnostic reference levels for diagnostic and interventional $\mathrm{X}$-ray procedures in Germany: update and handling. Rofo 191:739-751

10. European Society of Radiology, Paulo G, Damilakis J, et al (2020) Diagnostic reference levels based on clinical indications in computed tomography: a literature review. Insights Imaging 11. https://doi. org/10.1186/s13244-020-00899-y
11. Rehani MM (2015) Limitations of diagnostic reference level (DRL) and introduction of acceptable quality dose (AQD). Br J Radiol 88: 20140344

12. Vañó E, Miller DL, Martin CJ et al (2017) ICRP Publication 135: diagnostic reference levels in medical imaging. Ann ICRP 46:1144

13. Samei E, Järvinen H, Kortesniemi M et al (2018) Medical imaging dose optimisation from ground up: expert opinion of an international summit. J Radiol Prot 38:967-989

14. Wildberger JE, Prokop M (2020) Hounsfield's legacy. Investig Radiol 55:556-558

15. Marrero JA, Ahn J, Reddy RK (2014) ACG clinical guideline: the diagnosis and management of focal liver lesions. Am J Gastroenterol 109:1328-1347

16. Solomon J, Marin D, Roy Choudhury K, Patel B, Samei E (2017) Effect of radiation dose reduction and reconstruction algorithm on image noise, contrast, resolution, and detectability of subtle hypoattenuating liver lesions at multidetector CT: filtered back projection versus a commercial model-based iterative reconstruction algorithm. Radiology 284:777-787

17. Barrett HH, Myers KJ, Hoeschen C, Kupinski MA, Little MP (2015) Task-based measures of image quality and their relation to radiation dose and patient risk. Phys Med Biol 60:R1-R75

18. Barrett HH, Myers KJ (2004) Foundations of image science. WileyInterscience, Hoboken

19. Racine D, Viry A, Becce F et al (2017) Objective comparison of high-contrast spatial resolution and low-contrast detectability for various clinical protocols on multiple CT scanners. Med Phys 44: e153-e163

20. Racine D, Ryckx N, Ba A et al (2018) Task-based quantification of image quality using a model observer in abdominal CT: a multicentre study. Eur Radiol 28:5203-5210

21. Viry A, Aberle C, Racine D et al (2018) Effects of various generations of iterative $\mathrm{CT}$ reconstruction algorithms on low-contrast detectability as a function of the effective abdominal diameter: a quantitative task-based phantom study. Phys Med 48:111-118

22. Robinson PJ, Arnold P, Wilson D (2003) Small "indeterminate" lesions on CT of the liver: a follow-up study of stability. Br J Radiol 76:866-874

23. BIPM (2008) Evaluation of measurement data - guide to the expression of uncertainty in measurement. JCGM. Available via https://www.bipm.org/utils/common/documents/jegm/JCGM $100 \_2008$ E.pdf. Accessed 24 Mar 2021

24. Zhang Y, Leng S, Yu L et al (2014) Correlation between human and model observer performance for discrimination task in CT. Phys Med Biol 59:3389-3404

25. Racine D, Ott JG, Ba A, Ryckx N, Bochud FO, Verdun FR (2016) Objective task-based assessment of low-contrast detectability in iterative reconstruction. Radiat Prot Dosim 169:73-77

26. Ott JG, Ba A, Racine D, Viry A, Bochud FO, Verdun FR (2017) Assessment of low contrast detection in $\mathrm{CT}$ using model observers: developing a clinically-relevant tool for characterising adaptive statistical and model-based iterative reconstruction. Z Med Phys 27: 86-97

27. Wunderlich A, Noo F, Gallas BD, Heilbrun ME (2015) Exact confidence intervals for channelized Hotelling observer performance in image quality studies. IEEE Trans Med Imaging 34:453-464

28. Brankov JG (2013) Evaluation of the channelized Hotelling observer with an internal-noise model in a train-test paradigm for cardiac SPECT defect detection. Phys Med Biol 58:7159-7182

29. Ba A, Abbey CK, Baek J et al (2018) Inter-laboratory comparison of channelized hotelling observer computation. Med Phys 45: 3019-3030

30. Verdun FR, Racine D, Ott JG et al (2015) Image quality in CT: from physical measurements to model observers. Phys Med 31: 823-843 
31. Jendeberg J, Geijer H, Alshamari M, Cierzniak B, Liden M (2017) Size matters: The width and location of a ureteral stone accurately predict the chance of spontaneous passage. Eur Radiol 27:47754785

32. Burgess AE (2011) Visual perception studies and observer models in medical imaging. Semin Nucl Med 41:419-436

33. Gang GJ, Lee J, Stayman JW et al (2011) Analysis of Fourierdomain task-based detectability index in tomosynthesis and conebeam CT in relation to human observer performance. Med Phys 38: 1754-1768

34. Monnin P, Bosmans H, Verdun FR, Marshall NW (2016) A comprehensive model for quantum noise characterization in digital mammography. Phys Med Biol 61:2083-2108

35. Boone JM, Brink JA, Edyvean S et al (2012) Report 87. J ICRU. https://doi.org/10.1093/jicru/ndt006

36. Miéville FA, Gudinchet F, Brunelle F, Bochud FO, Verdun FR (2013) Iterative reconstruction methods in two different MDCT scanners: physical metrics and 4-alternative forced-choice detectability experiments - a phantom approach. Phys Med 29:99-110

37. Sharp P, Barber DC, Brown DG et al (1996) Report 54. J ICRU. https://doi.org/10.1093/jicru/os28.1.Report54

38. Bujila R, Fransson A, Poludniowski G (2017) Practical approaches to approximating MTF and NPS in CT with an example application to task-based observer studies. Phys Med 33:16-25

39. Zhang Y, Smitherman C, Samei E (2017) Size-specific optimization of CT protocols based on minimum detectability. Med Phys 44: 1301-1311

40. McCollough CH, Yu L, Kofler JM et al (2015) Degradation of CT low-contrast spatial resolution due to the use of iterative reconstruction and reduced dose levels. Radiology 276:499-506
41. Mileto A, Guimaraes LS, McCollough CH, Fletcher JG, Yu L (2019) State of the art in abdominal CT: the limits of iterative reconstruction algorithms. Radiology 293:491-503

42. Fletcher JG, Yu L, Fidler JL et al (2017) Estimation of observer performance for reduced radiation dose levels in CT. Acad Radiol 24:876-890

43. Solomon J, Ba A, Bochud F, Samei E (2016) Comparison of lowcontrast detectability between two CT reconstruction algorithms using voxel-based 3D printed textured phantoms. Med Phys 43: 6497-6506

44. Monnin P, Sfameni N, Gianoli A, Ding S (2018) Optimal slice thickness for object detection with longitudinal partial volume effects in computed tomography. J Appl Clin Med Phys 18(1):251259

45. Perisinakis K, Damilakis J, Tzedakis A, Papadakis A, Theocharopoulos N, Gourtsoyiannis N (2007) Determination of the weighted CT dose index in modern multi-detector CT scanners. Phys Med Biol 52:6485-6495

46. Boone JM (2007) The trouble with CTDI100: the trouble with CTDI100. Med Phys 34:1364-1371

47. Weir VJ, Zhang J (2019) Technical note: using linear and polynomial approximations to correct IEC CTDI measurements for a wide-beam CT scanner. Med Phys 46:5360-5365

Publisher's note Springer Nature remains neutral with regard to jurisdictional claims in published maps and institutional affiliations. 